\title{
Soft-collinear resummation in deeply virtual Compton scattering
}

\section{T. Altinoluk}

Departamento de Física de Partículas and IGFAE, Universidade de Santiago de Compostela, E-15782 Santiago de Compostela, Galicia-Spain

Email: tolga.altinoluk@usc.es

\section{B. Pire}

CPhT, École Polytechnique, CNRS, F-91128 Palaiseau, France

Email: pire@cpht.polytechnique.fr

\section{Szymanowski}

National Centre for Nuclear Research (NCBJ), Warsaw, Poland

Email: lech.szymanowski@fuw.edu.pl

\section{S. Wallon*}

LPT, Université Paris-Sud, CNRS, 91405, Orsay, France \&

UPMC Univ. Paris 06, faculté de physique, 4 place Jussieu, 75252 Paris Cedex 05, France

E-mail: walloneth.u-psud.fr

We derive an all order resummation formula for the deeply virtual Compton scattering amplitude, which takes into account soft-collinear gluon exchanges in the partonic (quark gluon) amplitude. We identify at each order of the perturbative series the diagrams responsible in a specific gauge to the leading contributions. An exponentiated expression results in a simple closed expression valid in the vicinity of the boundary regions between characteristic domains of the kinematical inner variables. This first example of soft-collinear resummation in exclusive reactions stabilizes the perturbative expansion which is crucial for a trustful extraction of generalized parton distributions from experimental data.

XXI International Workshop on Deep-Inelastic Scattering and Related Subject - DIS2013,

22-26 April 2013

Marseille, France

\footnotetext{
${ }^{*}$ Speaker.
} 


\section{Introduction}

While perturbative calculations are widely used in quantum field theory, their summation is always a formidable task, unreachable but in the simplest, not to say most simplistic, occurences. From elementary particle to atomic and to solid state physics, resummation techniques have been developped to go beyond a fixed order perturbation estimate through the sampling and evaluation of an infinite class of diagrams shown to dominate in a limited kinematical region for some given observables. The result of such procedures is often an exponentiated factor as in the famous Sudakov case [1], $\exp \left(-K g^{2} \log ^{2} z\right)$, where $g$ is the coupling constant and $z$ is the ratio of two different characteristic scales, which governs both QED and QCD calculations of exclusive form factors. Here we obtain for a specific case of exclusive scattering amplitude a resummed result of the form $\cosh (K g \log z)$ where $z$ is a momentum fraction. To our knowledge, this form never previously emerged in field theoretic calculations. The process that we focus on is the most studied case of a class of reactions - exclusive hard hadronic processes - which are under intense experimental investigation. The result presented here provides an important stepping-stone for further developments enabling a consistent extraction of the quantities describing the 3-dimensional structure of the proton.

In the collinear factorization framework the scattering amplitude for exclusive processes such as deeply virtual Compton scattering (DVCS) has been shown [2] to factorize in specific kinematical regions, provided a large scale controls the separation of short distance dominated partonic subprocesses and long distance hadronic matrix elements, the generalized parton distributions (GPDs) [3]. The amplitude for the DVCS process

$$
\gamma^{(*)}(q) N(p) \rightarrow \gamma\left(q^{\prime}\right) N^{\prime}\left(p^{\prime}\right),
$$

with a large virtuality $q^{2}=-Q^{2}$, factorizes in terms of perturbatively calculable coefficient functions $C\left(x, \xi, \alpha_{s}\right)$ and GPDs $H(x, \xi, t)$, where the scaling variable in the generalized Bjorken limit is the skewness $\xi$ defined as $\xi=\frac{Q^{2}}{\left(p+p^{\prime}\right) \cdot\left(q+q^{\prime}\right)}$. The calculation of first order perturbative corrections to the partonic amplitude has shown that terms of order $\frac{\log ^{2}(\xi \pm x)}{x \pm \xi}$ play an important role in the region of small $(x \pm \xi)$ i.e. in the vicinity of the boundary between the domains where the QCD evolution equations of GPDs take distinct forms (the so-called ERBL and DGLAP domains). We scrutinize these regions and demonstrate that they are dominated by soft fermion and gluon propagation. This explains why they can be exponentiated using quasi-eikonal techniques. The present report is a short version of Ref. [4], with a few investigations of phenomenological issues added.

\section{Full analysis}

\subsection{Lessons from the DVCS amplitude at NLO.}

Let us begin with the discussion of the NLO corrections to the amplitude for DVCS (1.1). After proper renormalization, the quark contribution to the symmetric part of the factorized Compton scattering amplitude reads

$$
\mathscr{A}^{\mu v}=g_{T}^{\mu v} \int_{-1}^{1} d x\left[\sum_{q}^{n_{F}} T^{q}(x) H^{q}(x, \xi, t)\right],
$$


where the quark coefficient function $T^{q}$ reads [5]

$$
\begin{aligned}
T^{q}= & C_{0}^{q}+C_{1}^{q}+C_{\text {coll }}^{q} \log \frac{Q^{2}}{\mu_{F}^{2}}, \\
C_{0}^{q}= & e_{q}^{2}\left(\frac{1}{x-\xi+i \varepsilon}-(x \rightarrow-x)\right), \\
C_{1}^{q}= & \frac{e_{q}^{2} \alpha_{S} C_{F}}{4 \pi(x-\xi+i \varepsilon)}\left\{\log ^{2}\left(\frac{\xi-x}{2 \xi}-i \varepsilon\right)-9\right. \\
& \left.-3 \frac{\xi-x}{\xi+x} \log \left(\frac{\xi-x}{2 \xi}-i \varepsilon\right)\right\}-(x \rightarrow-x) .
\end{aligned}
$$

The first (resp. second) terms in Eqs. (2.3) and (2.4) correspond to the $s$-channel (resp. $u$-channel) class of diagrams. One goes from the $s-$ channel to the $u$-channel by the interchange of the photon attachments. Since these two contributions are obtained from one another by a simple $(x \leftrightarrow-x)$ interchange, we now restrict mostly to the discussion of the former class of diagrams.

\subsection{Main steps of our analysis}

We start our analysis with the observation that in the same spirit as for evolution equations, the extraction of the soft-collinear singularities which dominate the amplitude in the limit $x \rightarrow \pm \xi$ is made easier if one uses the light-like gauge $p_{1} \cdot A=0$ with $p_{1}=q^{\prime}$. We argue that in this gauge the amplitude is dominated by ladder-like diagrams, see Fig. 1. In our analysis we expand any momentum in the Sudakov basis $p_{1}, p_{2}$, as $k=\alpha p_{1}+\beta p_{2}+k_{\perp}$, where $p_{2}$ is the light-cone direction of the two incoming and outgoing partons $\left(p_{1}^{2}=p_{2}^{2}=0,2 p_{1} \cdot p_{2}=s=Q^{2} / 2 \xi\right)$. In this basis, $q_{\gamma^{*}}=p_{1}-2 \xi p_{2}$.

We now restrict our study to the limit $x \rightarrow+\xi$. The dominant kinematics is given by a strong ordering both in longitudinal and transverse momenta, according to

$$
\begin{gathered}
x \sim \xi \gg\left|\beta_{1}\right| \sim|x-\xi| \gg\left|x-\xi+\beta_{1}\right| \sim\left|\beta_{2}\right| \gg \cdots \\
\cdots \gg\left|x-\xi+\beta_{1}+\beta_{2}+\cdots+\beta_{n-1}\right| \sim\left|\beta_{n}\right|, \\
\left|k_{\perp 1}^{2}\right| \ll\left|k_{\perp 2}^{2}\right| \ll \cdots \ll\left|k_{\perp n}^{2}\right| \ll s \sim Q^{2}, \\
\left|\alpha_{1}\right| \ll \cdots \ll\left|\alpha_{n}\right| \ll 1 .
\end{gathered}
$$

This ordering is related to the fact that the dominant double logarithmic contribution for each loop arises from the region of phase space where both soft and collinear singularities manifest themselves. When $x \rightarrow \xi$ the left fermionic line is a hard line, from which the gluons are emitted in an eikonal way (which means that these gluons have their all four-components neglected in the vertex w.r.t. the momentum of the emitter), with an ordering in $p_{2}$ direction and a collinear ordering. For the right fermionic line, eikonal approximation is not valid, since the dominant momentum flow along $p_{2}$ is from gluon to fermion, nevertheless the collinear approximation can still be applied.

When computing the coefficient functions, one faces both UV and IR divergencies. On the one hand, the UV divergencies are taken care of through renormalization, which manifest themselves by a renormalization scale $\mu_{R}$ dependency. On the other hand, the IR divergencies remain, but factorization proofs at any order for DVCS justify the fact that they can be absorbed inside the generalized parton distributions and result in finite coefficient functions. In our study, we are 


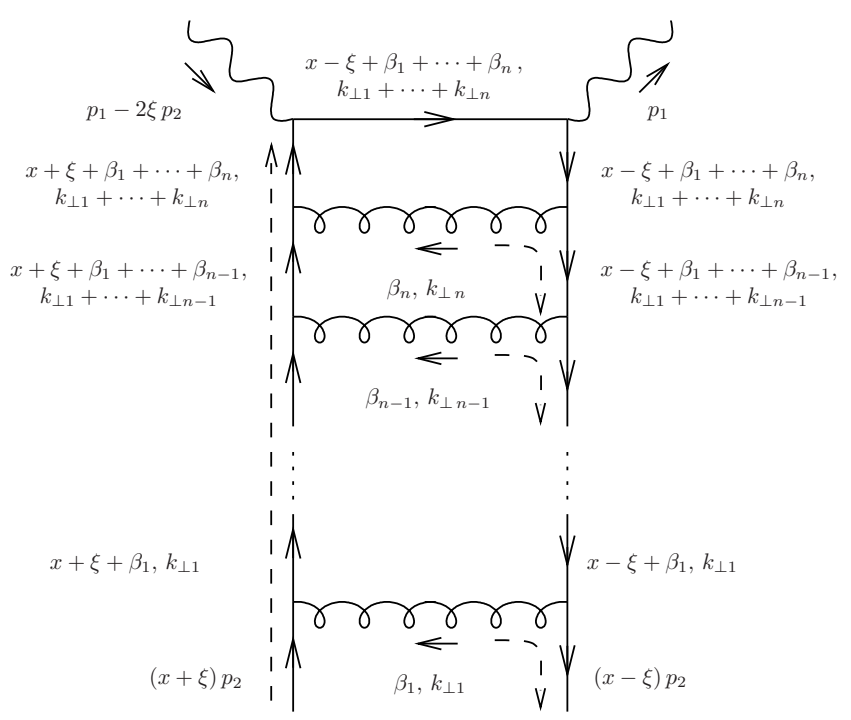

Figure 1: The ladder diagrams which contribute in the light-like gauge to the leading $\alpha_{s}^{n} \log ^{2 n}(\xi-x) /(x-\xi)$ terms in the perturbative expansion of the DVCS amplitude. The $p_{2}$ and $\perp$ momentum components are indicated. The dashed lines show the dominant momentum flows along the $p_{2}$ direction.

only interested into finite parts. Thus, using dimensional regularization, in a factorization scheme like $\overline{M S}$, any scaleless integral can be safely put to zero although it contains both UV and IR divergencies. Following this line of thought, we can thus safely deal with DVCS on a quark for our resummation purpose.

Finally, the issue related to the $i \varepsilon$ prescription in Eq. (2.4) is solved by computing the coefficient function in the unphysical region $\xi>1$. After analytical continuation to the physical region $0 \leq \xi \leq 1$, the final result is then obtained through the shift $\xi \rightarrow \xi-i \varepsilon$.

For further analysis, $K_{n}$ denotes the contribution of a $n$-loop ladder to the coefficient function

$$
K_{n}=-\frac{i}{4} e_{q}^{2}\left(-i C_{F} \alpha_{s} \frac{1}{(2 \pi)^{2}}\right)^{n} I_{n} .
$$

Let us sketch the main steps of the derivation of $I_{n}$ (see Ref. [4] for details).

\subsection{The ladder diagram at order $\alpha_{s}$}

A careful analysis [4] shows that the one-loop diagrams of Fig. 2 are not dominant for $x \rightarrow \xi$. Thus, we concentrate on the box diagram, see Fig. 1 with $n=1$. Starting from the dominant part of the numerator of the Born term which is $\theta=-2 \not p_{1}$, the numerator of the box diagram is

$$
\operatorname{tr}\left\{\not p_{2} \gamma^{\mu}\left[k+(x-\xi) \not p_{2}\right] \not \theta\left[k+(x+\xi) \not p_{2}\right] \gamma^{v}\right\} d^{\mu v}
$$

In the limit $x \rightarrow \xi$, while the left fermionic line is hard with a large $p_{2}$ momentum, the gluonic line is soft with respect to the left fermionic line. So we perform soft gluon approximation in the numerator by taking $k+(x+\xi) p_{2} \rightarrow(x+\xi) p_{2}$. The dominant contribution comes from the residue of the gluonic propagator. Thus, the numerator of the on-shell gluon propagator, $d^{\mu \nu}$, is expressed in terms of transverse polarizations, i.e. $d^{\mu \nu} \approx-\sum_{\lambda} \varepsilon_{(\lambda)}^{\mu} \varepsilon_{(\lambda)}^{v}$. 


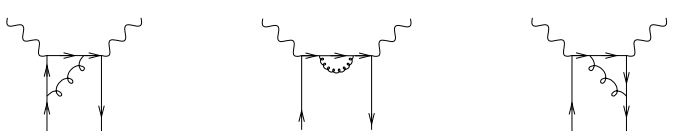

Figure 2: The one-loop diagrams which do not contribute to the leading $\frac{\log ^{2}(x-\xi)}{x-\xi}$ terms in the $p_{1} \cdot A=0$ light-like gauge.

The Sudakov decomposition of the gluon polarization vectors in the light-like $p_{1} \cdot A=0$ gauge

$$
\varepsilon_{(\lambda)}^{\mu}=\varepsilon_{\perp(\lambda)}^{\mu}-2 \frac{\varepsilon_{\perp(\lambda)} \cdot k_{\perp}}{\beta s} p_{1}^{\mu}
$$

allows us to define an effective vertex for the gluon and outgoing quark through the polarization sum

$$
\sum_{\lambda} \varepsilon_{\perp(\lambda)} \cdot k_{\perp} \varepsilon_{(\lambda)}^{\mu}=-k_{\perp}^{\mu}+2 \frac{k_{\perp}^{2}}{\beta s} p_{1}^{\mu} .
$$

The numerator, $(\mathrm{Num})_{1}$, is $\alpha$-independent and reads

$$
\begin{aligned}
& \frac{-4(x+\xi)}{\beta} \operatorname{tr}\left\{\not p_{2}\left(k_{\perp}-2 \frac{k_{\perp}^{2}}{\beta s} \not p_{1}\right)\left[k+(x-\xi) \not p_{2}\right] \not p_{1}\right\} \\
& =-4(x+\xi) s \frac{2 k_{\perp}^{2}}{\beta}\left[1+\frac{2(x-\xi)}{\beta}\right] .
\end{aligned}
$$

We emphasize that the term $(x+\xi) / \beta$ arises from the eikonal emission from the left fermionic line while the expression inside the $[\cdots]$ accounts for the fact that the gluon is not soft from the point of view of the right fermion (in an eikonal treatment it would reduce to $(x-\xi) / \beta$ ). We now calculate the integral over the gluon momentum $k$, using dimensional regularization $\int d^{d} k \rightarrow$ $\frac{s}{2} \int d \alpha d \beta d^{d-2} \underline{k},\left(k_{\perp}^{2}=-\underline{k}^{2}\right)$. The Cauchy integration of the gluonic pole which gives the dominant contribution reads

$$
I_{1}=-\left.2 \pi i \frac{s}{2} \int_{0}^{\xi-x} \frac{d \beta}{s \beta} \int_{0}^{\infty} d^{d-2} \underline{k} \frac{(N u m)_{1}}{L_{1}^{2} R_{1}^{2} S^{2}}\right|_{\alpha=\frac{k^{2}}{\beta s}}
$$

with the denominators $L_{1}^{2}=-\underline{k}^{2}+\alpha(\beta+x+\xi) s, R_{1}^{2}=-\underline{k}^{2}+\alpha(\beta+x-\xi) s, S^{2}=-\underline{k}^{2}+(\beta+x-$ $\xi) s$ and $k^{2}=-\underline{k}^{2}+\alpha \beta s$. The relevant region of integration corresponds to small $|\beta+x-\xi|$. The $\beta$ and $\underline{k}$ integrations results in our final one-loop expression

$$
I_{1}=-\frac{4}{x-\xi} \frac{2 \pi i}{2 !} \log ^{2}(\lambda(x-\xi))
$$

where we kept only the most singular terms in the $x \rightarrow \xi$ region and have no control of the value of $\lambda$ within our approximation. To fix $\lambda$, we match our approximated one-loop result with the full one-loop result (2.4). This amounts to cut the $\underline{k}^{2}$ integral at $Q^{2}$. The $i \varepsilon$ term is included according to the same matching. This leads to

$$
I_{1}=-\frac{4}{x-\xi+i \varepsilon} \frac{2 \pi i}{2 !} \log ^{2}\left(\frac{\xi-x}{2 \xi}-i \varepsilon\right) .
$$

A similar expression holds for the $u$-channel diagram in the limit $x \rightarrow-\xi$. 


\subsection{The ladder diagram at order $\alpha_{s}^{2}$}

Let us examine the next order in the perturbative expansion. There are many diagrams contributing but it can be shown that in the chosen gauge, the double box diagram dominates, and moreover that the relevant region of integration is where there is a strong ordering between $k_{2 \perp}$ and $k_{1 \perp}$, i.e. $k_{2 \perp} \gg k_{1 \perp}$. Using the same arguments as in the one-loop case, one simplifies the numerator for this diagram, after taking advantage of strong $k_{i \perp}$ ordering, as

$$
\begin{aligned}
(N u m)_{2}= & -4 s(x+\xi)^{2}\left\{\frac{2 k_{\perp 1}^{2}}{\beta_{1}}\left[1+\frac{2(x-\xi)}{\beta_{1}}\right]\right\} \\
& \times\left\{\frac{2 k_{\perp 2}^{2}}{\beta_{2}}\left[1+\frac{2\left(\beta_{1}+x-\xi\right)}{\beta_{2}}\right]\right\} .
\end{aligned}
$$

The denominators of fermionic propagators at the gluonic poles with $\alpha_{i}=-\frac{\underline{k}_{i}}{\beta_{i}}$ read (Fig. 1)

$$
\begin{aligned}
& L_{1}^{2}=\alpha_{1}(x+\xi) s, R_{1}^{2}=-\underline{k}_{1}^{2}+\alpha_{1}\left(\beta_{1}+x-\xi\right) s, \\
& L_{2}^{2}=\alpha_{2}(x+\xi) s, R_{2}^{2}=-\underline{k}_{2}^{2}+\alpha_{2}\left(\beta_{1}+\beta_{2}+x-\xi\right) s, \\
& S^{2}=-\underline{k}_{2}^{2}+\left(\beta_{1}+\beta_{2}+x-\xi\right) s .
\end{aligned}
$$

Using Cauchy integration over $\alpha_{1}$ and $\alpha_{2}$, we see that the dominant contribution comes from the residues corresponding to the two gluon propagators, and reads

$$
\begin{aligned}
& I_{2} \approx \int_{0}^{\xi-x} d \beta_{1} \int_{0}^{\xi-x-\beta_{1}} d \beta_{2} \int_{0}^{\infty} d^{d-2} \underline{k}_{2} \int_{0}^{\underline{k}_{2}^{2}} d^{d-2} \underline{k}_{1} \\
& \times \frac{4 s(2 \pi i)^{2}}{x-\xi} \frac{1}{\beta_{1}+x-\xi} \frac{1}{k_{1}^{2}} \frac{1}{\underline{k}_{2}^{2}} \frac{1}{\underline{k}_{2}^{2}-\left(\beta_{1}+\beta_{2}+x-\xi\right) s} .
\end{aligned}
$$

The integrals over $\underline{k}_{1}$ and $\underline{k}_{2}$ are performed by assuming that the singularities are regularized within dimensional regularization. Taking into account the fact that a scaleless integral vanishes, we get the following formula

$$
I_{2} \approx-\frac{4}{x-\xi+i \varepsilon} \frac{(2 i \pi)^{2}}{4 !} \log ^{4}\left(\frac{\xi-x}{2 \xi}-i \varepsilon\right)
$$

The $i \varepsilon$ and the $2 \xi$ factor in the argument of the logarithm are fixed by hand, extending the matching used at the one-loop level. These prescriptions are not needed for the resummation of $\left[\alpha_{s} \log ^{2}\left(\frac{\xi-x}{2 \xi}-\right.\right.$ $i \varepsilon)]^{n}$ terms which we want to exhibit and are beyond the accuracy of our estimate.

\subsection{The ladder diagram at order $\alpha_{s}^{n}$}

We now turn to the estimation of all $\log ^{2 n}(\xi-x)$ terms. We again assume the strong ordering $(2.5,2.6)$ in $k_{\perp}$ and $\beta$. The distribution of the poles generates nested integrals in $\beta_{i}$

$$
\int_{0}^{\xi-x} d \beta_{1} \int_{0}^{\xi-x-\beta_{1}} d \beta_{2} \cdots \int_{0}^{\xi-x-\beta_{1}-\cdots-\beta_{n-1}} d \beta_{n}
$$


The numerator for the $n^{\text {th }}$ order box diagram is obtained, by generalizing the argument for the two-loop case, as:

$$
\begin{aligned}
& (\text { Num })_{n}=-4 s(x+\xi)^{n} \frac{2 k_{\perp 1}^{2}}{\beta_{1}}\left[1+\frac{2(x-\xi)}{\beta_{1}}\right] \frac{2 k_{\perp 2}^{2}}{\beta_{2}} \\
& \times\left[1+\frac{2\left(\beta_{1}+x-\xi\right)}{\beta_{2}}\right] \cdots \frac{2 k_{\perp n}^{2}}{\beta_{n}}\left[1+\frac{2\left(\beta_{n-1}+\cdots+\beta_{1}+x-\xi\right)}{\beta_{n}}\right],
\end{aligned}
$$

and the denominators of propagators are, for $i=1 \cdots n$,

$$
\begin{aligned}
L_{i}^{2} & =\alpha_{i}(x+\xi) s \\
R_{i}^{2} & =-\underline{k}_{i}^{2}+\alpha_{i}\left(\beta_{1}+\cdots+\beta_{i}+x-\xi\right) s, \\
S^{2} & =-\underline{k}_{n}^{2}+\left(\beta_{1}+\cdots+\beta_{n}+x-\xi\right) s .
\end{aligned}
$$

Using dimensional regularization and omitting scaleless integrals, the integral $I_{n}$ reads:

$$
\begin{aligned}
I_{n}= & \int_{0}^{\xi} d \beta_{1}^{\xi} \cdots \int_{0}^{\xi-x-\cdots-\beta_{n-1}} d \beta_{n}^{\infty} d_{0}^{d-2} \underline{k}_{n} \cdots \int_{0}^{\underline{k}_{2}^{2}} d-2 \underline{k}_{1}(-1)^{n} \\
& \times \frac{4 s(2 \pi i)^{n}}{x-\xi} \frac{1}{\beta_{1}+x-\xi} \cdots \frac{1}{\beta_{1}+\cdots+\beta_{n-1}+x-\xi} \\
& \times \frac{1}{\underline{k}_{1}^{2}} \cdots \frac{1}{\underline{k}_{n}^{2}} \frac{1}{\underline{k}_{n}^{2}-\left(\beta_{1}+\cdots+\beta_{n}+x-\xi\right) s} .
\end{aligned}
$$

The integrals over $\underline{k}_{1} \cdots \underline{k}_{n}$ are performed similarly as in the one- and two-loop cases, leading to

$$
I_{n}=-\frac{4}{x-\xi+i \varepsilon} \frac{(2 \pi i)^{n}}{(2 n) !} \log ^{2 n}\left(\frac{\xi-x}{2 \xi}-i \varepsilon\right),
$$

where the matching condition introduced in the one-loop case is extended to $n$-loops.

\section{Result}

\subsection{The resummed formula}

Based on the results Eqs. $(2.15,2.19,2.24)$, one can build the resummed formula for the complete amplitude. Combining Eqs. $(2.8,2.24)$, we get

$$
\begin{aligned}
& \sum_{n=0}^{\infty} K_{n}=\frac{e_{q}^{2}}{x-\xi+i \varepsilon} \cosh \left[D \log \left(\frac{\xi-x}{2 \xi}-i \varepsilon\right)\right] \\
& =\frac{1}{2} \frac{e_{q}^{2}}{x-\xi+i \varepsilon}\left[\left(\frac{\xi-x}{2 \xi}-i \varepsilon\right)^{D}+\left(\frac{\xi-x}{2 \xi}-i \varepsilon\right)^{-D}\right] .
\end{aligned}
$$

In the absence of a next to leading logarithmic calculation, the minimal and most natural resummed formula which has the same $O\left(\alpha_{s}\right)$ expression as the full NLO result, reads, with $D=\sqrt{\frac{\alpha_{S} C_{F}}{2 \pi}}$,

$$
\begin{aligned}
& C^{\mathrm{res}}=\frac{e_{q}^{2}}{x-\xi+i \varepsilon}\left\{\cosh \left[D \log \left(\frac{\xi-x}{2 \xi}-i \varepsilon\right)\right]\right. \\
& \left.-\frac{D^{2}}{2}\left[9+3 \frac{\xi-x}{x+\xi} \log \left(\frac{\xi-x}{2 \xi}-i \varepsilon\right)\right]\right\}-(x \rightarrow-x) .
\end{aligned}
$$




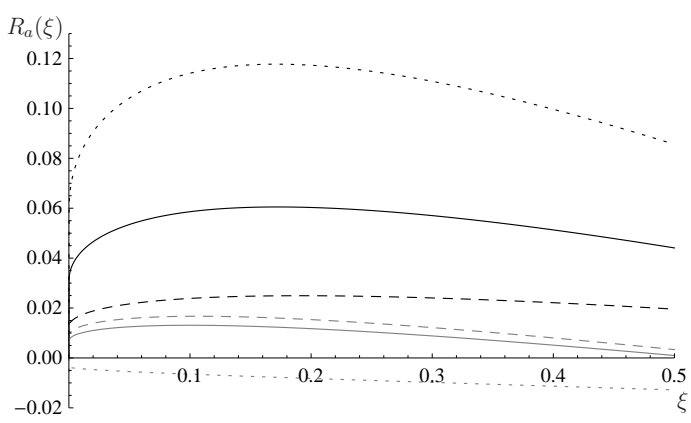

Figure 3: The real (black upper curves) and imaginary (grey lower curves) parts of the correction $R_{a}(\xi)$ defined by Eq. (3.3) with $a=1$ (solid), $a=1 / 2$ (dotted) and $a=2$ (dashed).

\subsection{Phenomenological perspectives}

To quantify the effect of our resummation procedure, we need to convolute the resummed coefficient function with a GPD. If we blindly perform the integral in the whole $x$-range, we find a result that differs from the NLO result by less than one per cent (with the model [6] and $\alpha_{s} \simeq 0.3$ ). This hints to a stabilized perturbative expansion around $x= \pm \xi$, in the sense that when convoluted with reasonable GPDs the resummed amplitude is nicely fitted by the NLO result. In order to respect the domain of applicability of our resummation procedure, we calculate the effect of restricting the use of our formula to a narrow $x$-range around $\pm \xi$, with a typical width $a \gamma$ defined through $|D \log (\gamma /(2 \xi))|=1$. The theoretical uncertainty is evaluated by taking various values of $a$. A more precise treatment of this uncertainty would require, as usual, to go beyond the leading logarithmic approximation. In Fig. 3 we plot the ratio $R_{a}(\xi)$ of the correction to the modulus of the NLO Compton form factor, defined as:

$$
R_{a}(\xi)=\frac{\left[\int_{\xi-a \gamma}^{\xi+a \gamma}+\int_{-\xi-a \gamma}^{-\xi+a \gamma}\right] d x\left(C^{\mathrm{res}}-C_{0}-C_{1}\right) H(x, \xi, 0)}{\left|\int_{-1}^{1} d x\left(C_{0}+C_{1}\right) H(x, \xi, 0)\right|} .
$$

The fact that $R_{a}(\xi)$ does not exceed a few per cent, proves that the stabilization of the perturbative expansion is a robust feature of our resummation procedure.

\section{Conclusions}

We have demonstrated that resummation of soft-collinear gluon radiation effects can be performed in hard exclusive reactions amplitudes. The resulting formula for coefficient function stabilizes the perturbative expansion, which is crucial for a trustful extraction of GPDs from experimental data. A related expression should emerge in various reactions, such as the crossed case of timelike Compton scattering [7] and exclusive meson electroproduction. We did not study neither the effects of the running of $\alpha_{s}$ nor the case of gluon GPD contributions which, although they begin at order $\alpha_{s}$ are expected to be important in the small $\xi$ regime to be accessible at high energies [8]. It has been customary to perform resummation in terms of Mellin moments [9]. Here, conformal Mellin moments may be more appropriate, and we will address this question in future work.

We thank A. H. Mueller, G. Sterman, O. V. Teryaev, J. Wagner, for useful discussions and correspondence. This work is partly supported by the ANR "Partons" grant, the Polish Grant NCN No DEC- 2011/01/D/ST2/03915, the Joint Research Activity "Study of Strongly Interacting 
Matter" (HadronPhysics3, Grant Agreement n.283286) under the $7^{\text {th }}$ Framework Programme of the European Community, the European Research Council grant HotLHC ERC-2001- StG-279579, the Ministerio de Ciencia e Innovacíon of Spain grants FPA2009-06867-E, Consolider-Ingenio 2010 CPAN CSD2007-00042 and FEDER.

\section{References}

[1] V. V. Sudakov, Sov. Phys. JETP 3, 65 (1956) [Zh. Eksp. Teor. Fiz. 30, 87 (1956)].

[2] D. Müller et al., Fortsch. Phys. 42, 101 (1994); A. V. Radyushkin, Phys. Lett. B 380, 417 (1996); X. D. Ji and J. Osborne, Phys. Rev. D 58, 094018 (1998) and Phys. Rev. D 57, 1337 (1998); J. Collins and A. Freund, Phys. Rev. D59, 074009 (1999).

[3] K. Goeke, M. V. Polyakov and M. Vanderhaeghen, Prog. Part. Nucl. Phys. 47, 401 (2001); M. Diehl, Phys. Rept. 388, 41 (2003); A. V. Belitsky and A. V. Radyushkin, Phys. Rept. 418, 1 (2005).

[4] T. Altinoluk, B. Pire, L. Szymanowski and S. Wallon, JHEP 1210, 49 (2012).

[5] A. V. Belitsky and D. Mueller, Phys. Lett. B 417, 129 (1998); A. V. Belitsky et al., Phys. Lett. B 474, 163 (2000); B. Pire, L. Szymanowski and J. Wagner, Phys. Rev. D 83, 034009 (2011).

[6] S. V. Goloskokov and P. Kroll, Eur. Phys. J. C 50, 829 (2007).

[7] D. Mueller, B. Pire, L. Szymanowski and J. Wagner, Phys. Rev. D 86 (2012) 031502.

[8] D. Boer et al., arXiv:1108.1713 [nucl-th]; J. L. Abelleira Fernandez et al., J. Phys. G 39, 075001 (2012); B. Pire, L. Szymanowski and J. Wagner, Phys. Rev. D 79 (2009) 014010.

[9] A. Bassetto, M. Ciafaloni, G. Marchesini and A. H. Mueller, Nucl. Phys. B 207, 189 (1982); G. F. Sterman, Nucl. Phys. B 281, 310 (1987); S. Catani and L. Trentadue, Nucl. Phys. B 327, 323 (1989). 